\section{Introducción de la vacuna conjugada contra Hib en Chile y Uruguay}

\author{
Mauricio Landaverde, ${ }^{1}$ \\ José Luis Di Fabio, ${ }^{1}$ \\ Gloria Ruocco, ${ }^{2}$ Irene Leal ${ }^{3}$ \\ y Ciro de Quadros ${ }^{1}$
}

En algunos países, la enfermedad invasora causada por Haemophilus influenzae tipo b (Hib) ha sido prácticamente eliminada gracias a la vacunación. Sin embargo, en gran parte del mundo en desarrollo las meningitis y neumonías causadas por esas bacterias siguen siendo una causa importante de morbilidad y mortalidad infantiles, así como de elevados costos de hospitalización.

Desde 1990 se cuenta con vacunas conjugadas, seguras y eficaces. Los frecuentes informes en la literatura médica que destacan los logros de la salud pública en los países donde dichas vacunas han sido introducidas ha motivado al Programa Especial para Vacunas e Inmunización (SVI) de la OPS a recomendar su introducción como parte de los esquemas regulares de vacunación en todos aquellos países en los que la relación costo-beneficio sea ventajosa y no ponga en riesgo la sostenibilidad del programa.

En este artículo se presentan las experiencias que dos países de América Latina, Chile y Uruguay, acumularon en el proceso de introducir la vacuna como medida de salud pública para la vacunación ordinaria de los niños. Durante el proceso, al igual que con toda nueva experiencia, se encontraron obstáculos que podrían haberse evitado de haberse contado con información detallada sobre la introducción de la vacuna en otros países. Al transmitir la valiosa información así obtenida, esperamos facilitar el quehacer y la toma de decisiones de los países que en el futuro decidan proteger a sus niños vacunándolos contra las enfermedades causadas por Hib.

\section{MATERIALES Y MÉTODOS}

Entre junio y agosto de 1997, se recolectó en Chile y Uruguay la información solicitada en el cuestionario "Introducción de vacunas conjugadas contra el Hib: monitoreo de países que recientemente adoptaron la vacunación de rutina con la vacuna Hib conjugada", preparado por la Iniciativa en pro de la Vacunación Infantil.

Ese instrumento, dirigido a obtener información de los mandos técnicos y políticos involucrados en la introducción de la vacuna contra Hib en sus países, agrupa la información en las siguientes categorías generales: 1) cómo se tomó la decisión de introducir la vacuna y las razones para incluirla en el esquema de vacunación regular de los niños del país; 2) cómo y en qué condiciones se introdujo la 
vacuna (registro sanitario, formulación, sola o combinada, esquema); 3) inmunogenicidad y efecto en la enfermedad; 4) impacto sobre el Programa Ampliado de Inmunización, y 5) resumen de las principales lecciones aprendidas.

La parte que concierne al proceso que culminó en la decisión de introducir la vacuna y las razones para incluirla en el esquema de vacunación regular de los niños del país procuraba identificar los elementos esenciales que dieron lugar a la decisión definitiva. Se entrevistó mediante preguntas abiertas a los responsables de la vigilancia epidemiológica, los programas de inmunizaciones y los laboratorios de salud pública en los ministerios de salud respectivos para determinar quién o quiénes iniciaron el proceso, quiénes fueron sus principales proponentes y defensores, cuál fue la información epidemiológica analizada, cuáles fueron los principales argumentos a favor o en contra, quién o quiénes tomaron la decisión final y qué información sobre experiencias previas hubiese facilitado el proceso.

En lo que se refiere a las condiciones para introducir la vacuna, el cuestionario investigaba aspectos relacionados con el registro sanitario de la vacuna en el país: su presencia o ausencia en el mercado privado, las presentaciones disponibles y la seleccionada, la formulación escogida y el esquema o los esquemas de vacunación utilizados, los criterios tomados en consideración, los costos de adquisición y los elementos o conocimientos que hubiesen facilitado la tarea a los responsables de esas determinaciones.

En cuanto a los aspectos de inmunogenicidad y efectividad, el cuestionario indagaba acerca de estudios nacionales de inmunogenicidad efectuados antes de la introducción de la vacuna, así como de estudios que se estuviesen realizando en ese momento. Se solicitaba también la información epidemiológica disponible sobre la enfermedad (incidencia, mortalidad, series hospitalarias, estudios de base poblacional, series de resultados de laboratorio y tasas), tanto la acumulada previamente como la obtenida después de introducir la vacuna.

Además se analizaron el sistema de vigilancia epidemiológica y los planes para medir el impacto de la introducción de la vacuna. En este sentido, se estudió el impacto que tuvo la vacuna sobre el Programa Nacional de Inmunizaciones analizando la información sobre las tareas y costos que significó en las áreas de presupuesto, cadena de frío, capacitación, cobertura, efectos adversos asociados con la vacuna, reacciones del sector público y de los usuarios, y datos facilitados a la comunidad y a las sociedades científicas.

La parte final del cuestionario correspondiente a las lecciones aprendidas preguntaba sobre los principales éxitos obtenidos, las mayores dificultades encontradas; cómo podría facilitarse el pro- ceso y las consideraciones que todo gerente debería tener en cuenta para garantizar el éxito del programa. En esta parte también se hacía hincapié en cuáles lecciones surgidas de la experiencia les gustaría transmitir a quienes estuvieran considerando introducir la vacuna contra Hib como actividad regular de vacunación para los niños de sus países.

\section{RESULTADOS}

A continuación se presentan los datos obtenidos de Chile y Uruguay con los cuestionarios descritos.

\section{Chile}

Razones para la introducción de la vacuna Hib. En 1995, Chile y los demás países del Cono Sur tuvieron una epidemia de meningitis meningocócica que despertó en la comunidad, los medios de comunicación y las sociedades científicas relacionadas con la medicina una intensa preocupación por tomar medidas preventivas contra "las meningitis". En ese contexto, el Ministerio de Salud creó una Comisión de Expertos para analizar la situación, y sobre la base de su recomendación, el Ministro de Salud tomó la decisión de introducir en Chile la vacuna contra $H$. influenzae tipo b, con la intención de disminuir la incidencia de meningitis y otras enfermedades invasoras.

La Comisión de Expertos tomó en consideración los antecedentes citados en la literatura; los estudios sobre eficacia, respuesta serológica y efectividad realizados en el país; la epidemiología de la enfermedad; los análisis de impacto y costo beneficio; el informe técnico de factibilidad, y los costos logísticos y económicos que la introducción de la vacuna implicaría. Se analizó en especial la principal objeción presentada contra dicha medida: el costo alternativo de su introducción, es decir, la posibilidad de que dichos recursos pudiesen ser destinados a mejor uso dentro del campo de la salud.

La introducción de la vacuna como medida de salud pública, cuya aceptación y demanda habían sido ya parcialmente generadas por la presencia de dicho producto en el mercado privado, contó con el amplio respaldo de las sociedades de pediatría e infectología, y se hizo en seguimiento a un decreto ministerial y presidencial.

Introducción de la vacuna. La vacuna contra Hib fue registrada y estuvo disponible en el mercado privado desde 1992. Si bien no se cuenta con datos de la cantidad de niños vacunados o del número 
de dosis administradas en la práctica privada, se sabe que desde esa fecha hasta 1996, se vendieron 126365 dosis en el país.

El Programa de Inmunizaciones de Chile inició la vacunación contra Hib en julio de 1996, aplicando a las nuevas cohortes de niños menores de 1 año tres dosis, administradas a los 2, 4 y 6 meses de edad. La vacuna fue adquirida mediante licitación pública nacional y tuvo un costo promedio de US $\$ 4,50$ por dosis. Durante el primer año se adquirió en las siguientes presentaciones: liofilizada, líquida, en frascos de 1 y 10 dosis, como antígeno único y en combinación con otros. La compra de Hib dio lugar posteriormente a una donación de vacunas DPT por el laboratorio ganador de la licitación.

La vacuna contra Hib, al igual que las otras vacunas del Programa Ampliado de Inmunización (PAI), se aplica gratuitamente y los recursos para adquirirla en los próximos años han sido incorporados al presupuesto nacional.

Inmunogenicidad, efectividad e impacto. En Chile se ha informado sobre las enfermedades invasoras y la meningitis por Hib en numerosas publicaciones. Como aspectos sobresalientes vale la pena mencionar que, en el Área Metropolitana de Santiago entre 1985 y 1987, la incidencia anual de meningitis fue de 25,7 por 100000 menores de 5 años; $98 \%$ de los casos se registraron en menores de 2 años y $80 \%$, antes de los 18 meses de edad.
La vigilancia epidemiológica que se lleva a cabo en todo el país incluye a los prestadores de servicios públicos y privados y está basada en la notificación obligatoria de los casos. La vigilancia se reforzó a partir de enero de 1996, fecha en que se capacitó a los epidemiólogos y personal de laboratorio de todas las regiones del país, quienes a su vez adiestraron al personal de sus áreas de responsabilidad. El Instituto de Salud Pública, en su calidad de laboratorio de referencia nacional, desarrolló también un programa de transferencia de tecnología destinado a facilitar el diagnóstico local y la remisión correcta de las muestras positivas. Se establecieron una definición de caso y una ficha estándar de investigación, y se definieron conductos para el flujo de información, los cuales fueron incorporados al sistema nacional de vigilancia epidemiológica del país. Aunque sería aún prematuro determinar el impacto definitivo de la vacunación sobre la incidencia de la enfermedad, y pese a que actualmente la cobertura de la primera cohorte de menores de 1 año es de $92 \%$, ya comienza a notarse una disminución del número de casos (figura 1).

Impacto sobre el Programa de Inmunizaciones. Para introducir la vacuna contra Hib no fue necesario adquirir nuevo equipo de cadena de frío, ni incrementar los gastos relacionados con el almacenamiento y la distribución de vacunas. Las coberturas alcanzadas año tras año con las otras vacunas del Programa continúan manteniéndose por encima de

FIGURA 1. Casos de Haemophilus influenzae tipo b, Chile, 1995-1997

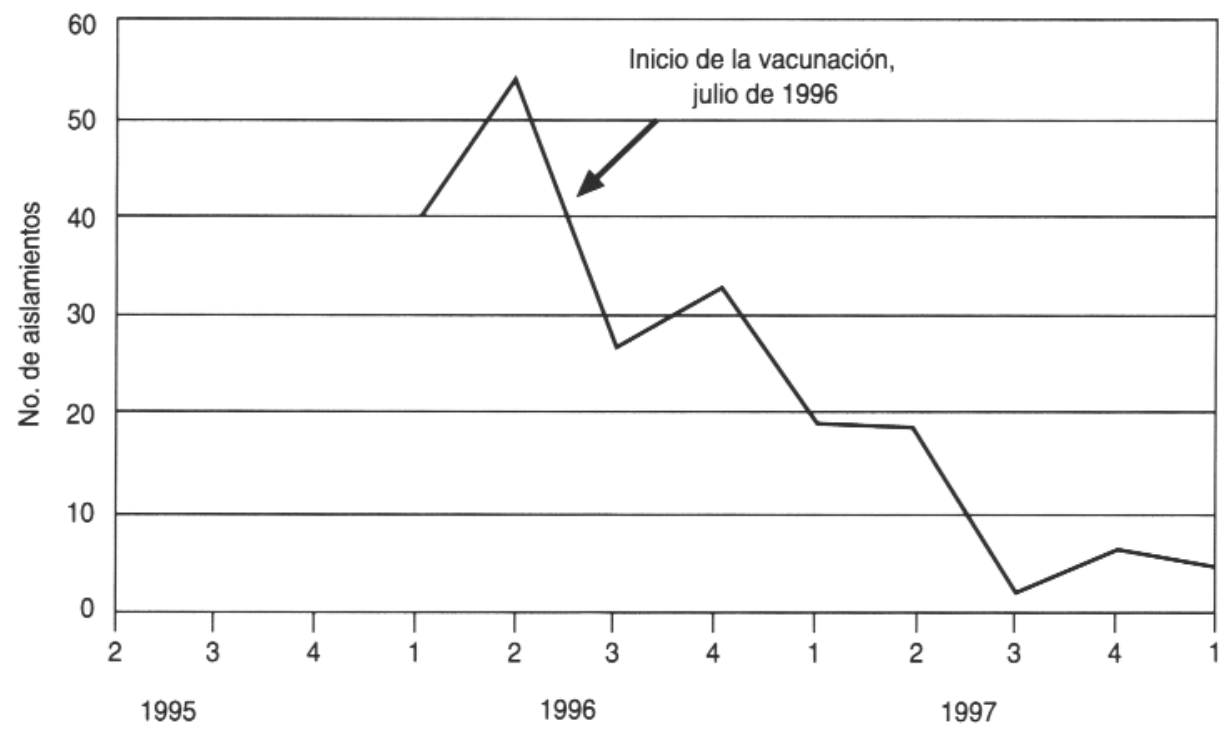


94\% sin que el agregar al esquema una nueva vacuna haya repercutido negativamente en los logros alcanzados. Las modificaciones efectuadas al carnet de vacunación, a las hojas de registro y al sistema de monitoreo de efectos adversos posvacunales no representaron gastos especiales. Los trabajadores de salud informaron de la nueva vacuna a la comunidad durante las visitas de las madres a los establecimientos de salud para vacunar a sus hijos. La introducción de la vacuna no se acompañó de una campaña de comunicación social. Durante el primer año de aplicación, se adquirieron 1030000 dosis, a un costo de US\$ 6,4 millones incluidos los impuestos nacionales de adquisición y los gastos administrativos. Ello representó para el país un incremento de $384 \%$ sobre los gastos del año anterior para todas las otras vacunas del Programa en los mismos rubros.

\section{Principales lecciones aprendidas}

- Las medidas tomadas para fortalecer el sistema de vigilancia epidemiológica debieron haberse iniciado con más anticipación. Ello hubiera permitido, antes de empezar la vacunación, conocer mejor la incidencia de la enfermedad causada por Hib, lo que habría facilitado la evaluación de los efectos de la vacuna.

- Los criterios para seleccionar una versión de la vacuna para el Programa debieron establecerse con mayor precisión. Con ello se habría facilitado el proceso de capacitación en el servicio y evitado adquirir vacunas en frascos multidosis que dieron lugar a bastante desperdicio.

- La decisión política, la adecuada justificación técnica para su introducción, el apoyo de las sociedades científicas y la aceptación por la comunidad constituyeron condiciones básicas que facilitaron la introducción de la vacuna y han contribuido a mejorar el prestigio del Programa de Inmunizaciones.

\section{Uruguay}

Razones para la introducción de la vacuna contra Hib. En 1994, en respuesta a crecientes demandas de la comunidad, el Ministerio de Salud Pública del Uruguay introdujo en el país la vacuna contra Hib. Dichas demandas fueron generadas por la epidemia de meningitis meningocócica que se presentó en esos años en el Uruguay y países vecinos y que contribuyó a concienciar a la población sobre la posibilidad de prevenir las "meningitis". En vista de ello, la Dirección de Epidemiología y la Comisión Nacional de Inmunizaciones, con el apoyo de las Socieda- des Uruguayas de Infectología y Pediatría, impulsaron la propuesta que llevó al Ministro de Salud a tomar la decisión de introducir la vacuna en el país.

La Comisión Nacional de Inmunizaciones basó su recomendación de introducir la vacuna contra Hib en la información disponible sobre la incidencia de las enfermedades causadas por ese agente, estudios efectuados en la comunidad y resultados etiológicos disponibles. También tuvo en cuenta la principal preocupación que dicha medida ocasionaba desde el punto de vista de las finanzas del sector, el alto costo de la vacuna y la factibilidad de garantizar su financiación en años venideros, situación que quedó resuelta y garantizada por decreto presidencial. La vacuna se aplica a todos los niños del país de manera gratuita, tanto por prestadores de servicios públicos como privados.

Introducción de la vacuna. Se desconoce el total de dosis aplicadas o de niños protegidos por medio del sistema de la práctica privada desde que la vacuna fue registrada e introducida en el país en 1993.

El Programa de Inmunizaciones inició la vacunación con Hib en agosto de 1994. La inmunización se centró en las nuevas cohortes de niños menores de 1 año, con tres dosis administradas a los 2, 4 y 6 meses de edad, y una dosis de refuerzo aplicada entre los 12 y 15 meses de edad. A los niños captados entre los 7 y 11 meses de edad se les aplican dos dosis, con intervalos de 2 meses y un refuerzo administrado entre los 12 y 15 meses. A los niños captados entre 1 y 4 años se les aplica una sola dosis.

El costo promedio de la vacuna ascendió a US\$ 6,00 por dosis. Se adquirió mediante licitación pública nacional y durante el primer año se usaron las siguientes presentaciones: liofilizada, en frascos de 1 y 10 dosis, y como antígeno único. La adquisición de la vacuna no estuvo ligada a donaciones de los laboratorios productores.

Inmunogenicidad, efectividad e impacto. En el Uruguay, las meningitis, y entre ellas las causadas por $\mathrm{Hib}$, han sido de notificación obligatoria desde 1961, y objeto de vigilancia del sistema nacional, tal como se muestra en la figura 2. En ella puede también apreciarse el impacto que tuvo sobre la incidencia a nivel nacional la aplicación de la vacuna, que durante el año de introducción se aplicó a 87\% de los niños entre 1 y 4 años, y se inició la vacunación de las nuevas cohortes de recién nacidos. La información sobre cobertura se obtiene mediante un sistema de registro personalizado en el cual se inscribe a los recién nacidos de cada nueva cohorte. 
FIGURA 2. Casos de Haemophilus influenzae tipo b, Uruguay, 1993-1997

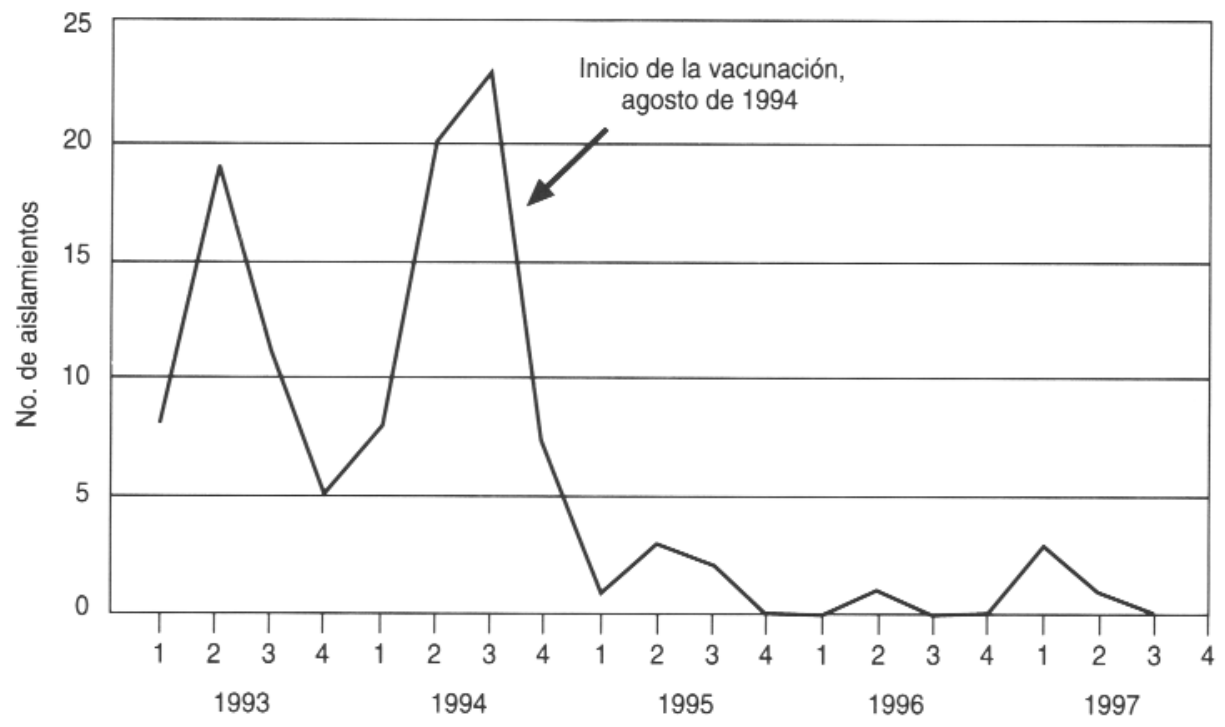

La vigilancia epidemiológica, activa en todo el país, fue reforzada después de haberse introducido la vacuna como medida de salud pública. Incluye a los prestadores de servicios públicos y privados y está basada en la notificación obligatoria de los casos.

Durante el período de capacitación del personal clínico y de laboratorio en los hospitales se hizo hincapié en mejorar la detección de casos, manejar adecuadamente las muestras de sangre y líquido cefalorraquídeo y continuar las medidas de control de contactos que ya venían realizándose desde 1991.

Impacto sobre el Programa de Inmunizaciones. Para introducir la vacuna contra Hib no fue necesario adquirir nuevo equipo de cadena de frío, ni incrementar los gastos relacionados con la distribución de vacunas. La cobertura alcanzada año tras año para todas las vacunas del Programa continúan manteniéndose por encima de $94 \%$. Las modificaciones del carnet de vacunación y del sistema de registro de dosis no implicó gastos adicionales.

La necesidad de información expresada por la comunidad fue satisfecha por los trabajadores de salud durante las visitas de las madres a los establecimientos de salud para vacunar a sus hijos. La introducción de la vacuna no fue acompañada de una campaña educativa específica dirigida a la comunidad, pero contó con amplia difusión en los medios de comunicación y se celebraron gran número de eventos dirigidos a las sociedades científicas del país.
La proporción de pérdidas fue de $12 \%$ en 1995, incluidas todas las vacunas. Aún no se ha evaluado la proporción de pérdidas correspondiente a la vacuna contra Hib ni su impacto sobre el total de dosis perdidas por todo el Programa.

\section{Principales lecciones aprendidas}

- El hecho de contar con un buen sistema de vigilancia y con adecuada información epidemiológica sobre la enfermedad facilitó la obtención de apoyo por parte de los profesionales de la salud y permitió argüir ante el poder ejecutivo la pertinencia de introducir la vacuna.

- La presión ejercida por la comunidad para que se tomaran medidas contra las "meningitis" fue decisiva para introducir esta disposición de salud pública que ha tenido un impacto evidente.

- La obtención de decretos legales que garanticen la continuidad de la vacunación fue un acierto que debe tenerse en consideración al implantar medidas que deben mantenerse por largo tiempo.

\section{DISCUSIÓN}

Si bien existen matices que permiten establecer diferencias entre ambos países, en términos generales tanto en Chile como en Uruguay la introducción de la vacuna contra Hib siguió una secuencia que permite establecer pautas de referencia y extraer lec- 
ciones que pueden ser aprovechadas por los países a punto de iniciar un proceso semejante.

En la introducción de la vacuna Hib en ambos países, la importancia que la prevención de las "meningitis" había adquirido en la opinión pública, así como el conocimiento de las sociedades científicas y los ministerios de salud de que había una buena vacuna disponible fueron factores decisivos. Aunados a la experiencia previa con la vacuna en el mercado privado, esos factores hicieron que los países tomaran la decisión de establecer comisiones o grupos de trabajo, los cuales, tras analizar las relaciones costo-beneficio, emitieron recomendaciones a las autoridades nacionales responsables de tomar la decisión final.

Por su parte, las autoridades nacionales de salud no solo apoyaron las recomendaciones de esos grupos científicos sino que además consiguieron el compromiso de los niveles políticos más altos, los cuales garantizaron que la introducción de la vacuna al Programa se hiciera de manera permanente y con financiamiento garantizado para los años venideros.

Las vacunas se adquirieron mediante licitaciones públicas nacionales en las que el precio por dosis osciló entre $\$ U S 4,50$ y 6,00. Debido a la falta de experiencia al establecer las bases de las licitaciones, en algunas circunstancias se adquirieron vacunas en presentaciones de dosis múltiples, liofilizadas o combinadas, que ocasionaron dificultades de aplicación y un incremento en la proporción de vacunas perdidas después de abiertos los frascos.

Las vacunas contra Hib fueron incorporadas al esquema nacional de vacunación y aplicadas durante los servicios de atención ordinaria en todo el territorio nacional, de forma gratuita, en ambos países. Se otorgó prioridad de vacunación a las nuevas cohortes de menores de 1 año, para quienes se programaron dosis a los 2, 4 y 6 meses de edad. En el Uruguay, también los niños captados entre los 7 y 11 meses de edad recibieron dos dosis y los mayores de 1 pero menores de 5 años, una sola dosis y se programó una cuarta dosis de refuerzo entre los 12 y 15 meses de edad. Se hizo un gran esfuerzo para garantizar que todos los menores de 5 años recibieran como mínimo una dosis de vacuna. Durante el primer año de aplicación, $87 \%$ de los niños uruguayos menores de 5 años habían completado sus esquemas de vacunación. En Chile, la proporción de menores de 1 año que completó el esquema fue de $90 \%$, aproximadamente.

El impacto de la vacunación sobre la incidencia de las meningitis causadas por Hib ha sido evidente en ambos países (véanse las figuras 1 y 2), mas cabe notar que ha sido más claro en Uruguay, donde la introducción de la vacuna se dirigió simultáneamente a todo el grupo de menores de 5 años. A ello hay que agregar que, en Chile, la instalación de un verdadero sistema de vigilancia de $H$. influenzae tipo b solo se hizo 6 meses antes de la introducción de la vacuna, circunstancia que podría de alguna manera hacer parecer la incidencia previa menos alta de lo que en realidad era en ese momento.

A excepción del incremento en el presupuesto destinado a la adquisición de vacunas y la capacitación de personal en vigilancia epidemiológica, la introducción de la vacuna contra Hib no ha significado una sobrecarga especial para los programas de inmunización de esos países en lo que refiere a gastos o esfuerzos de logística, manejo de información, cadena de frío, capacitación del personal operativo o información a las madres sobre los beneficios de la vacunación.

\section{CONCLUSIONES}

La introducción de la nueva vacuna contra Hib en Chile y Uruguay ha sido una experiencia positiva tanto para los trabajadores de salud y las asociaciones de profesionales como para la población infantil beneficiaria y sus padres. Estos últimos han visto satisfechas sus demandas de contar con una medida preventiva contra las "meningitis" y realizadas sus aspiraciones de poder ofrecer a sus hijos una vacuna que ya estaba disponible en el mercado privado y a la cual pocos tenían acceso.

Los gerentes de los programas de inmunizaciones enfrentaron las dificultades propias de quienes tienen que tomar medidas preventivas sin contar con toda la información necesaria al respecto. Sus experiencias pueden ser aprovechadas en otros países para lograr no solo el respaldo de las asociaciones de profesionales, sino también el apoyo de las autoridades políticas responsables de tomar las decisiones finales. La formación de comités de expertos a los que se encomendó la tarea de analizar la posible conveniencia de introducir la vacuna facilitó el apoyo de las asociaciones de profesionales y permitió a las autoridades de salud respaldar su decisión con la opinión de un grupo independiente.

La necesidad de contar con un adecuado sistema de vigilancia epidemiológica se hizo evidente, tanto para apoyar el proceso de toma de decisiones como para mostrar el impacto de la medida tomada. Dicho sistema debe ponerse en marcha en lo posible mucho antes de introducir la vacuna. Además, deben evaluarse cuidadosamente los criterios para seleccionar las especificaciones de la vacuna que se va a usar, ya que debido a su alto costo la proporción de dosis desechadas debe mantenerse en niveles cercanos a cero. Por otra parte, es indispensable obtener el compromiso político que garantice la compra de vacunas en años futuros, sin que ello sig- 
nifique restricciones en los recursos ya asignados al programa. La disponibilidad de mecanismos de compra a gran escala habría facilitado y acelerado en gran medida la decisión de introducir la vacuna.

Nota de la Redacción: Se espera que para 1999 la vacuna Hib esté incluida en el esquema de inmunización regular de $75 \%$ de los niños nacidos en América Latina y el Caribe.

\section{SYNOPSIS}

\section{Introducing Hib conjugate vaccine in Chile and Uruguay}

In some countries, the invasive disease caused by Haemophilus influenzae type $b$ (Hib) has been practically eliminated thanks to vaccination. However, in much of the developing world, meningitides and pneumonias caused by these bacteria continue to be a major cause of childhood morbidity and mortality, as well as high hospitalization costs. Because safe and effective conjugate vaccines are now available, the Special Program for Vaccines and Immunization of the Pan American Health Organization has recommended introducing them into the regular vaccination regimen of as many countries as possible. This has been done in Chile and Uruguay, where the Hib vaccine now forms part of the regular vaccination routine. When the vaccine was being introduced, both countries had difficulties they could have avoided if they had known of the experiences of other nations. Therefore, these two countries now offer the lessons they learned to other nations considering introducing the vaccine into their immunization programs. The most important lessons were to: strengthen the epidemiological surveillance system sufficiently in advance of introducing the vaccine; with the support of scientific societies, present the technical information that justifies introducing the vaccine; seek community backing and acceptance; precisely establish in advance the presentation and dosage of the vaccine that is most appropriate for the country; and be certain to have the political and legal decisions needed to ensure the continuity of Hib vaccination in the future.

\section{BIBLIOGRAFÍA}

1. Adams W, Deaver K, Cochi S, Plikaytis B, Zell E, Broome C, et al. Decline of childhood Haemophilus influenzae type $\mathrm{b}$ (Hib) disease in the Hib vaccine era. JAMA 1993; 269:221-226.

2. Booy R, Heath P, Slack M, Begg N, Moxon R. Vaccine failures after primary immunization with Haemophilus influenzae type b conjugate vaccine without booster. Lancet 1997; 349:1197-1202.

3. Booy R, Hodgson S, Carpenter L, Mayon-White R, Slack M, Macfarlane J, et al. Efficacy of Haemophilus influenzae type $\mathrm{b}$ conjugate vaccine PRP-T. Lancet 1994;344:362-366.

4. Camou T, Pallas C, Musser J, Hortal M. Molecular epidemiology of Haemophilus influenzae type $\mathrm{b}$ isolates from Uruguay. Rev Microbiol (São Paulo) 1996; 27:106-110.

5. Chávez A, Rojas C, Cohen L, Chadid J. Infecciones invasoras por Haemophilus influenzae $\mathrm{b}$. Rev Chile Infectol 1996;13(1):27-33.

6. Clements DA, Booy R, Dagan R, Gilbert GL, Moxon ER, Slack MP, et al. Comparison of the epidemiology and cost of Haemophilus influenzae type $\mathrm{b}$ disease in five Western countries. Pediatr Infect Dis J 1993; 12:362-367.
7. Ferreccio C, Ortiz E, Astroza L, Rivera C, Clemens J, Levine MM. A population-based retrospective assessment of the disease burden resulting from invasive Haemophilus influenzae in infants and young children en Santiago, Chile. Pediatr Infect Dis J 1990; 9:488-494.

8. Hortal M, Mogdasy C, Russi J, Deleón C, Suárez A. Microbial agents associated with pneumonia in children from Uruguay. Rev Infect Dis 1990;12:915-922.

9. Lagos R, Horwitz I, Toro J, San Martín O, Abrego P, Bustamante C, et al. Large scale postlicensure, selective vaccination of Chilean infants with PRP-T conjugate vaccine: practicality and effectiveness in preventing invasive Haemophilus influenzae type $b$ infections. Pediatr Infect Dis J 1996;15:216-222.

10. Levine OS, Ortiz E, Contreras R Lagos R, Vial P, Misraji A, et al. Cost-benefit analysis for the use of Haemophilus influenzae type b conjugate vaccine in Santiago, Chile. Am. J. Epidemiol. 1993;137:121-128.

11. Livartowski A, Boucher J, Detournay B, Reinert P. Cost-effectiveness evaluation of vaccination against
Haemophilus influenzae invasive diseases in France. Vaccine 1996; 14: 495-500.

12. Mulholland $K$, Hilton $S$, Adegbola R, Usen S, Oparaugo A, Omosigho C, et al. Randomised trial of Haemophilus influenzae type $\mathrm{b}$ tetanus protein conjugate for prevention of pneumonia and meningitis in Gambian infants. Lancet 1997;349:1191-1197.

13. Murphy $T$, White $K$, Pastor $P$, Gabriel L, Medley F, Granoff D, et al. Declining incidence of Haemophilus influenzae type $\mathrm{b}$ disease since introduction of vaccination. JAMA 1993; 269:246-248.

14. Peltola H. Haemophilus influenzae type $b$ disease and vaccination in Latin America and the Caribbean. Pediatr Infect Dis J 1997;16:780-787.

15. Peltola H, Kilpi T, Anttila M. Rapid disappearance of Haemophilus influenzae type $\mathrm{b}$ meningitis after routine childhood immunization with conjugate vaccines. Lancet 1992;340: 592-594.

16. Shapiro E, Ward J. The epidemiology and prevention of disease caused by Haemophilus influenzae type $\mathrm{b}$. Epidemiological Reviews 1991:13: 113-142. 